\title{
Microperimetric Biofeedback Training Improved Visual Acuity after Successful Macular Hole Surgery
}

\author{
Tomoko Ueda-Consolvo, Mitsuya Otsuka, Yumiko Hayashi, \\ Masaaki Ishida, and Atsushi Hayashi \\ Department of Ophthalmology, Graduate School of Medicine and Pharmaceutical Sciences, \\ University of Toyama, 2630 Sugitani, Toyama 930-0194, Japan \\ Correspondence should be addressed to Atsushi Hayashi; ahayashi@med.u-toyama.ac.jp
}

Received 4 October 2015; Revised 26 November 2015; Accepted 30 November 2015

Academic Editor: Enzo M. Vingolo

Copyright (c) 2015 Tomoko Ueda-Consolvo et al. This is an open access article distributed under the Creative Commons Attribution License, which permits unrestricted use, distribution, and reproduction in any medium, provided the original work is properly cited.

Purpose. To evaluate the efficacy of setting a preferred retinal locus relocation target (PRT) and performing Macular Integrity Assessment (MAIA) biofeedback training in patients showing insufficient recovery of best corrected visual acuity (BCVA) despite successful closure of an idiopathic macular hole (MH). Methods. Retrospective interventional case series. Nine eyes of 9 consecutive patients with the decimal BCVA of less than 0.6 at more than 3 months after successful MH surgery were included. A PRT was chosen based on MAIA microperimetry and the patients underwent MAIA biofeedback training. BCVA, reading speed, fixation stability, and 63\% bivariate contour ellipse area (BCEA) were evaluated before and after the training. Statistical analysis was carried out using paired Student's $t$-test. Results. PRT was chosen on the nasal side of the closed MH fovea in 8 patients. After the MAIA training, BCVA improved in all patients. The mean logMAR value of BCVA significantly improved from 0.33 to $0.12(p=0.007)$. Reading speed improved in all patients $(p=0.29)$, fixation stability improved in 5 patients $(p=0.70)$, and 63\% BCEA improved in 7 patients $(p=0.21)$, although these improvements were not statistically significant. Conclusion. MAIA biofeedback training improved visual acuity in patients with insufficient recovery of BCVA after successful MH surgery.

\section{Introduction}

Vision impairment and metamorphopsia are major symptoms of patients with an idiopathic macular hole $(\mathrm{MH})$. MH surgery has been improved since Kelly and Wendel first reported successful closure of MHs by use of pars plana vitrectomy with gas-fluid exchange [1]. A technique of internal limiting membrane (ILM) peeling improved visual outcomes and the closure rate in $\mathrm{MH}$ surgeries [2, 3]. Microincision vitrectomy surgery has shortened the operating time and improved patient comfort and visual recovery time [4]. Numerous studies have reported preoperative predictive factors for visual outcomes following $\mathrm{MH}$ surgery, including stage and size of $\mathrm{MH}$ [5], duration of symptoms [6], preoperative visual acuity [7], retinal sensitivity, fixation status [8], and optical coherence tomography (OCT) parameters such as minimum diameter of $\mathrm{MH}$, base hole diameter, the hole form factor, $\mathrm{MH}$ index, and inner segment/outer segment junction defect length [9]. These factors are not enough, however, to predict visual outcomes precisely. Visual outcomes are often worse than expected despite successful MH surgery.

Recently, foveal displacement following $\mathrm{MH}$ surgery has been reported [10-13]. After ILM peeling and gas tamponade, the retina is displaced toward the optic disc [10-13]. Ishida et al. showed that the ratio of retinal displacement in the temporal field was significantly correlated with the basal diameter of the $\mathrm{MH}$ [11]. We hypothesized that the foveal displacement might be one of the reasons for poor visual recovery after successful MH surgery. Helping patients to fix at the point of the best visual acuity after the closure of $\mathrm{MH}$ might improve their visual performances.

Biofeedback training by means of a microperimeter has been reported as an efficient method to improve the visual performance of patients with macular diseases such as age-related macular degeneration, Stargardt's disease, cone dystrophy, vitelliform dystrophy, and posttraumatic macular 


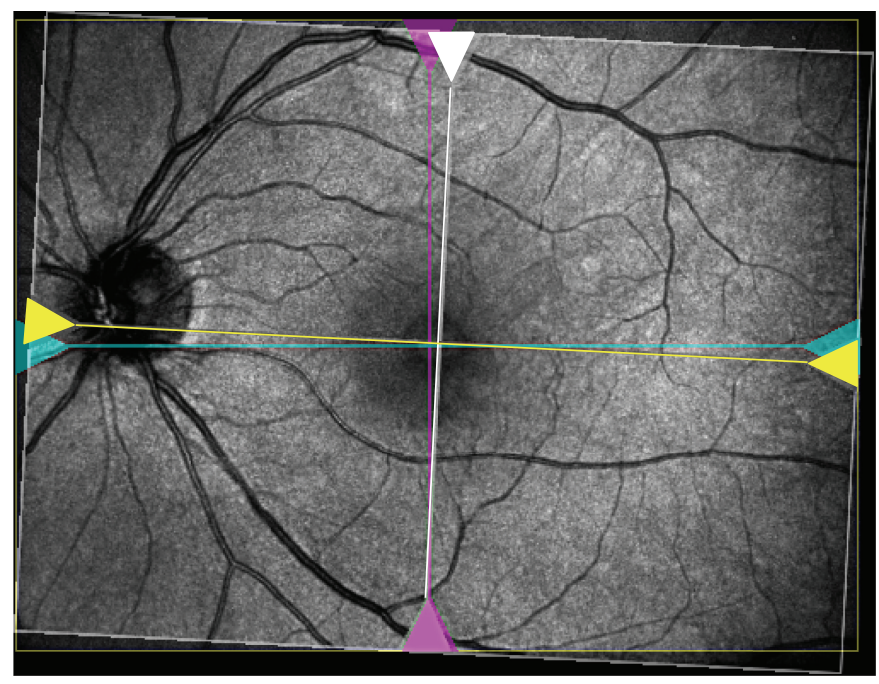

(a)

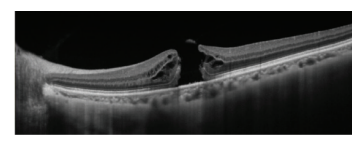

(b)

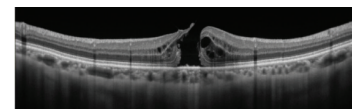

(c)

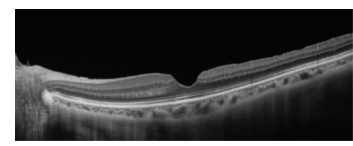

(d)

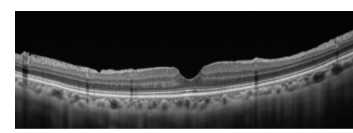

(e)

FIGURE 1: (a) Composite infrared image made by overlapping preoperative and postoperative images (case 6). Yellow line and white line indicate preoperative location of the horizontal and vertical scan (b, c). Blue line and pink line indicate postoperative location of the horizontal and vertical scan (d, e). Center of MH and fovea were identified by moving the location of scans (yellow, white, blue, and pink lines). The fovea has shifted toward the optic disc after MH surgery.

scar [14-16]. The training helped the patients stably fix their gaze at the preferred retinal locus relocation target (PRT) near the fovea.

In this study, we performed biofeedback training using a Macular Integrity Assessment (MAIA) microperimeter to provide an efficient PRT and to improve visual performance in patients who had insufficient visual recovery after successful closure of a $\mathrm{MH}$.

\section{Methods}

2.1. Inclusion and Exclusion Criteria. We recruited 9 eyes of 9 consecutive patients ( 4 men and 5 women), who had undergone $\mathrm{MH}$ surgery at Toyama University Hospital between May 2013 and June 2014. Inclusion criteria were (1) patients with idiopathic $\mathrm{MH}$, (2) patients in whom closure of $\mathrm{MH}$ was confirmed with optical coherence tomography (OCT) by the first MH surgery, (3) patients with visual acuity of 0.6 or less after 3 months of the MH surgery, (4) patients who could undergo OCT examinations with a single spectral domain OCT machine (RS-3000 Advance, NIDEK Co., Ltd., Aichi, Japan) before and after the $\mathrm{MH}$ surgery, and (5) patients who agreed with the MAIA training and were followed up for more than 3 months. Exclusion criteria were the presence of ocular complications that could affect visual performance, such as macular degeneration, rhegmatogenous retinal detachment, diabetic retinopathy, glaucoma, and corneal diseases.

2.2. Ophthalmic Examinations. All patients underwent comprehensive ophthalmologic examinations, including measurement of the decimal best corrected visual acuity (BCVA), reading speed test, intraocular pressure, slit-lamp biomicroscopy with a contact lens, OCT, and fixation stability test. Axial length was measured in all eyes preoperatively (OA-1000, Tomey, Aichi, Japan). The single spectral domain OCT machine (RS-3000 Advance) was used to evaluate tomographic features through the macula. The macula was scanned with the macula map mode of $9 \mathrm{~mm} \times 9 \mathrm{~mm}$ scan $(512 \times 128)$ or $9 \mathrm{~mm} \times 12 \mathrm{~mm}$ scan $(256 \times 128)$ by RS-3000 Advance. The basal diameter of each $\mathrm{MH}$ was measured with a caliper built in the software of the OCT machine.

All eyes underwent a standard pars plana vitrectomy with three 25-gauge ports. All patients underwent ILM peeling around the $\mathrm{MH}$ in the same manner. Sulfur hexafluoride gas (20\%) was used as a tamponade gas at the end of the surgery and the patients were asked to adopt face-down position for at least one hour after the surgery.

The distance of foveal displacement in each eye was measured after the closure of $\mathrm{MH}$ according to the methods of Kawano et al. [10]. Briefly, infrared fundus images were taken together with OCT images. The center of the $\mathrm{MH}$ was marked in the preoperative infrared fundus image by referencing OCT images (Figures $1(\mathrm{a}), 1(\mathrm{~b})$, and $1(\mathrm{c})$ ), and the length on the image was corrected with the axial length. Postoperatively, the center of foveal depression was similarly marked in the infrared fundus image by reference with OCT images within 3 months after the MH surgery (Figures 1(a), $1(\mathrm{~d})$, and $1(\mathrm{e}))$. The two marked infrared fundus images were overlapped manually (Figure 1(a)) and the distance of foveal displacement between the marks was measured using ImageJ software (National Institutes of Health, Bethesda, MD, available at http://imagej.nih.gov/ij/).

Reading speed was measured by reading Japanese words written in black on white background at a distance of $30 \mathrm{~cm}$ with appropriate refractive correction (MNRead-J, Handaya, 
TABLE 1: Preoperative clinical characteristics.

\begin{tabular}{lcccccc}
\hline Patient number & Sex & Age (years) & Stage & Duration of symptoms (months) & Basal diameter $(\mu \mathrm{m})$ & Preoperative decimal BCVA \\
\hline 1 & F & 64 & 3 & 1 & 571 & 015 \\
2 & M & 66 & 3 & Unknown & 483 & 0.2 \\
3 & F & 75 & 4 & 1.5 & 949 & 0.8 \\
4 & M & 70 & 3 & 1 & 457 & 0.15 \\
5 & M & 69 & 3 & 1 & 459 & 0.1 \\
6 & F & 65 & 3 & 2 & 588 & 0.15 \\
7 & F & 67 & 3 & 2 & 369 & 0.15 \\
8 & M & 64 & 3 & 1 & & 0.15 \\
9 & F & 64 & 3 & & & \\
\hline
\end{tabular}

BCVA: best corrected visual acuity.

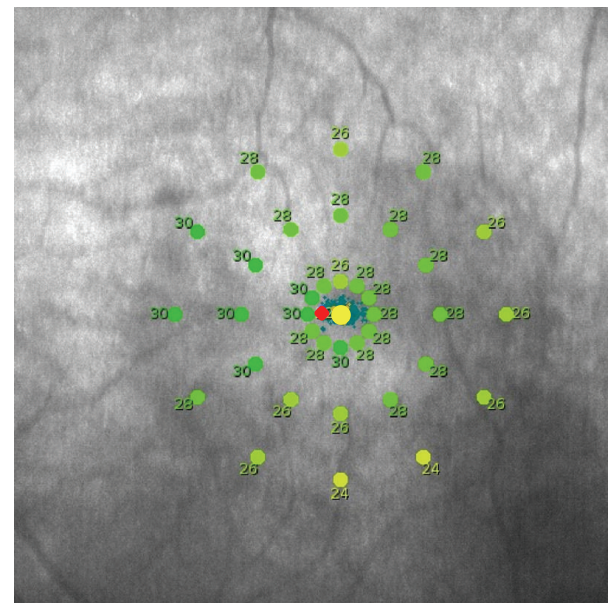

FIgURE 2: Each colored dot indicates retinal threshold sensitivity (case 8 ). We chose a region within the 2-degree ring that had the highest potential retinal sensitivity and set the new PRT (red dot) within that area.

Tokyo, Japan). Patients were asked to read the letters aloud as fast as possible without skipping any letters. The sentences contained high-frequency, nontechnical words. A fixation stability test was performed with an MAIA microperimeter (Topcon, Tokyo, Japan). The percentage of fixation points located within the 2-degree circle of the PRT was measured.

Sixty-three percent of bivariate contour ellipse area (BCEA) was also measured to evaluate fixation stability. The $63 \%$ BCEA is the elliptical area which encompasses $63 \%$ of fixation points during one fixation trial. A smaller BCEA correlates to more stable fixation. Square degree (Sqd) was used as the unit for the $63 \%$ BCEA.

2.3. Defining Preferred Retinal Locus Relocation Target (PRT). Before starting biofeedback training, macular threshold sensitivity and fixation stability were assessed with a MAIA microperimeter using an automated program. Retinal threshold sensitivity was displayed within a 10-degree range of the gravitational center of all fixation points. We chose a region within the 2 -degree ring that had the highest potential retinal sensitivity and set the new PRT within that area (Figure 2). If the retinal threshold sensitivity did not exhibit any difference within 2-degree ring, 6 points were screened as candidate PRTs and one point was selected according to fixation stability.

2.4. MAIA Biofeedback Training. MAIA biofeedback training was performed using a PRL training module for 10 minutes each session and repeated at least three times within three months. Before beginning the MAIA biofeedback training, the patients were asked to fix their gaze at the PRT by themselves according to an audio feedback program equipped with MAIA. This audio feedback program advised the patients whether or not they were getting closer to the PRT. All the procedures were checked by the examiner.

2.5. Statistical Analysis. All statistical analyses were carried out using JMP statistical discovery software (Version 9; SAS Institute, Cary, NC). Paired Student's $t$-test was used to compare the differences between values before and after the training. Spearman correlation coefficient was used to investigate correlations between 63\% BCEA and BCVA. Statistical significance was defined as $p<0.05$. The BCVA was measured with a Landolt $C$ chart in decimal units and converted to a logarithm of the minimum angle of resolution $(\log M A R)$ for statistical analysis.

\section{Results}

3.1. Characteristics of the Patients. Nine eyes of 9 patients were examined. The preoperative characteristics of the patients are listed in Table 1. The patients' ages ranged from 64 years old to 75 years old $(67.2 \pm 3.4$ years old, mean \pm standard deviation (SD)). The decimal BCVA before idiopathic $\mathrm{MH}$ surgery ranged from 0.1 to 0.8 . There were 8 eyes with stage 3 macular holes and 1 eye with stage 4 macular hole. In the preoperative OCT images, the basal diameter of the macular hole ranged from $369 \mu \mathrm{m}$ to $949 \mu \mathrm{m}$ (592 \pm $178 \mu \mathrm{m}$; mean $\pm \mathrm{SD}$ ). Anatomical closure of the $\mathrm{MH}$ was confirmed by OCT examination and no recurrence of $\mathrm{MH}$ occurred in any patients in this study. The mean distance of foveal displacement was $155.4 \pm 102.6 \mu \mathrm{m}$ (range: $35.7 \mu \mathrm{m}$ to $387.6 \mu \mathrm{m}$ ). Eight of the 9 eyes revealed displacement of the foveal center in the nasal direction and in the one eye the foveal center displaced in the temporal direction. 


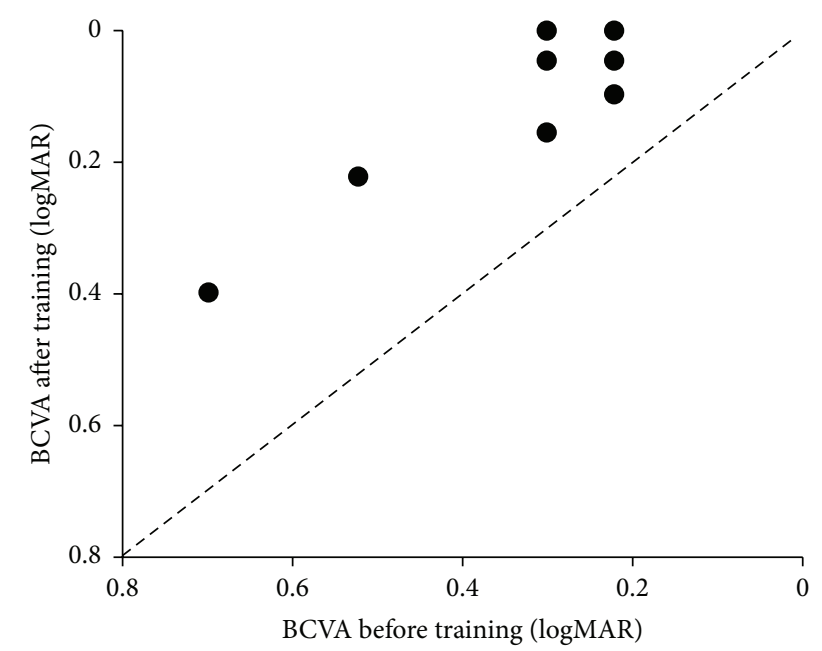

FIGURE 3: Scatter plot of best corrected visual acuity (BCVA) before and after MAIA biofeedback training.

The PRT in each patient was selected according to the sensitivity of the results of microperimetry and the patient's responses. The PRT was selected on the nasal side of the foveal center of the closed $\mathrm{MH}$ within 2 degrees in 8 of the 9 patients and in the other patient the PRT was chosen superior to the foveal center of the closed $\mathrm{MH}$ within 2 degrees. The patients started MAIA biofeedback training at 3 to 9 months (4.6 \pm 1.9 months) after the MH surgery (Table 2). When the patients wished to continue the MAIA biofeedback training after completing 3 sessions, we allowed them to continue it. Five patients ended MAIA training after 3 sessions, while 2 patients performed 4 sessions and 2 patients completed 5 sessions ( $3.7 \pm 0.8$ times; mean $\pm \mathrm{SD})$. The training period ranged from 1.5 to 4 months $(2.3 \pm 0.7$ months; mean $\pm S D)$ (Table 2).

3.2. Visual Acuity. BCVA improved in all patients after the MAIA biofeedback training (Figure 3). The mean logMAR value of BCVA was 0.33 at the baseline, but it significantly improved to 0.12 after 3 MAIA training sessions ( $p=0.007$ ) (Table 2). Five patients showed rapid improvement of BCVA after the initial MAIA training.

3.3. Reading Speed. The MAIA biofeedback training accelerated the reading speed in all patients. The reading speed before the training was $339 \pm 72$ words/min and slightly improved to $378 \pm 71$ words/min at the end of the training, a difference that was not statistically significant $(p=0.29)$ (Table 2).

3.4. Fixation Stability. Fixation stability was improved in five patients (patients 1, 2, 4, 5, and 6 in Table 2). In three patients (7-9 in Table 2), fixation stability was unchanged following the training. Two of those patients (patients 8 and 9 in Table 2) showed $100 \%$ fixation sensitivity before the start of MAIA training. In one patient (patient 3 in Table 2), fixation stability worsened. The mean fixation stability slightly

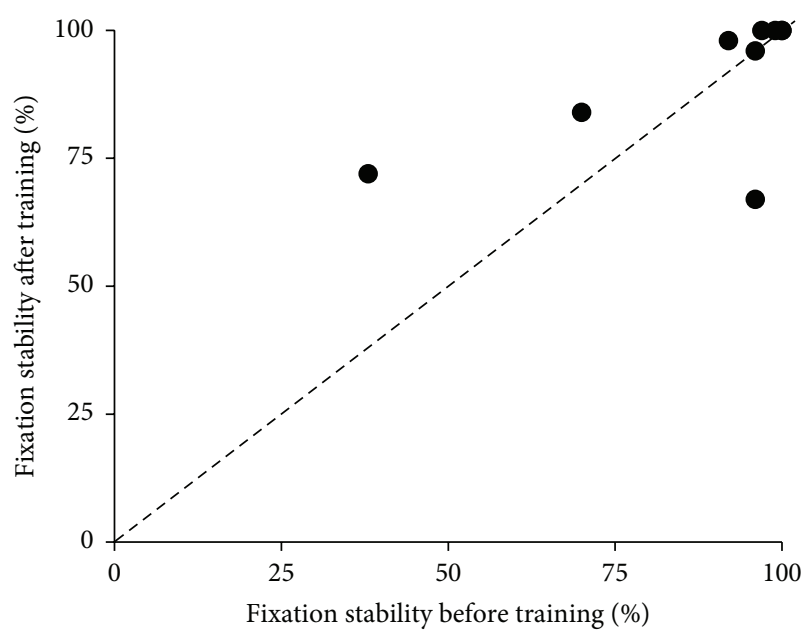

FIGURE 4: Scatter plot of fixation stability before and after MAIA biofeedback training.

improved from $88 \pm 20 \%$ before the training to $91 \pm 12 \%$ after the training, a difference that was not statistically significant $(p=0.70)$ (Table 2 and Figure 4).

The $63 \%$ BCEA was improved in 7 of 9 patients. In one patient the $63 \%$ BCEA was unchanged. The $63 \%$ BCEA was worsened in one patient. The mean 63\% BCEA improved from $0.96 \pm 0.91$ Sqd before the training to $0.42 \pm 0.49 \mathrm{Sqd}$ after the training, although it was not statistically significant $(p=0.21)$ (Table 2). There was no correlation between 63\% BCEA and BCVA before the training (Spearman correlation coefficients, $r=0.36 ; p=0.34)$ or after the training $(r=0.27$; $p=0.49$ ).

\section{Discussion}

The current study demonstrated that biofeedback training using MAIA microperimetry effectively improved the visual acuity of patients when the visual acuity had not fully recovered after successful closure of the $\mathrm{MH}$.

Microperimetric biofeedback training has been performed to improve visual performance of patients with several diseases such as age-related macular degeneration, Stargardt's disease, cone dystrophy, macular myopic degeneration, vitelliform dystrophy, and posttraumatic macular scars [14-16]. Patients with these diseases lost the fixation at the central fovea and were trained to fix at an extrafoveal area (PRT) to improve their visual acuity. In this study, we examined the possibility of biofeedback training to improve the BCVA and visual performances in patients with insufficient recovery of visual acuity after the successful closure of a MH.

One of the advantages using MAIA biofeedback training was that the patients could easily understand and repeat the training with the aid of the audio feedback informing them whether or not they were getting closer to the PRT. Sound perception increases the conscious attention of patients [17], and the increased attention helps the brain fix the PRT $[14,18]$. Vingolo et al. reported that audio feedback 


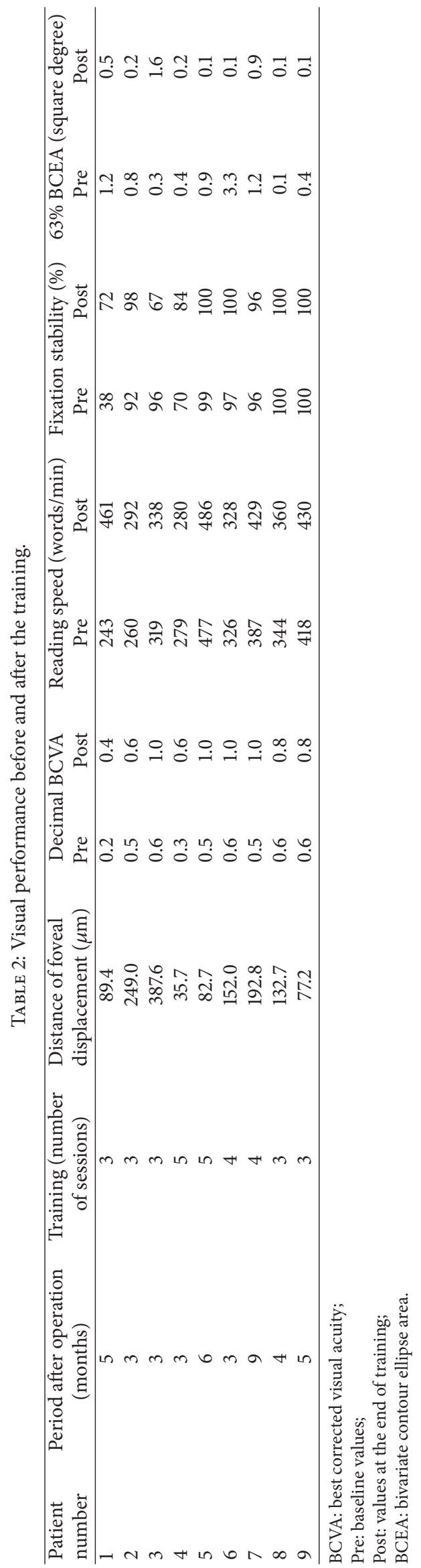


facilitated stimuli transmission between intraterinal neurons as well as between the retina and the brain and supports a "remapping phenomenon" [15]. The second advantage was that the examiner could select an appropriate PRT in the macula according to the results of the microperimetry and the patients could repeat the training at the same PRT every time.

Kawano et al. reported that the center of the macula area moved toward the optic disc an average distance of 0.1 disc diameter after vitrectomy with ILM peeling [10]. Ishida et al. showed that the ratio of the displacement of the temporal vessel was significantly correlated with the maximum size of the preoperative MH [11]. Nakagomi et al. showed that the postoperative fovea-to-disc distance $(3.82 \pm 0.34 \mathrm{~mm})$ was significantly shorter than the preoperative one (4.00 \pm $0.33 \mathrm{~mm}, p<0.0001)$ [13]. These results suggest that the center of the fovea shifted after successful MH surgery with ILM peeling in most patients. Foveal displacement following $\mathrm{MH}$ surgery might be one of the reasons for insufficient visual recovery after the closure of $\mathrm{MH}$, and introducing a PRT near the anatomical center of the fovea might result in better visual performance such as visual acuity, reading speed, and fixation stability for those patients. In our study, careful selection of a new PRT, followed by MAIA biofeedback training, improved visual acuity in all patients.

The mean distance of foveal displacement in this study did not differ from those in previous studies [10,11, 13]. Therefore, patients who showed insufficient recovery of visual acuity were not categorized into a specific group of patients. In addition, the correlation between the location of the PRT and the distance of foveal displacement was unclear. This might be due to the fact that the preferred retinal locus was located on any margin of the preoperative macular hole [19]. In this study, however, the PRT was located slightly to the nasal side of the foveal center in 8 patients (89\%). It appears that the fovea with the highest visual acuity might shift to the nasal side of the retina after successful closure of $\mathrm{MH}$.

Visual recovery after macular hole surgery sometimes requires more than 3 months. We cannot exclude the possibility that the visual acuity in our cases improved as part of the natural course during MAIA biofeedback training. In our investigation, however, BCVA immediately improved after the initial MAIA training in 5 of 9 patients and improved further after the completion of 3 MAIA training sessions.

The patients did not show a significant improvement of reading speed, fixation stability, nor 63\% BCEA after MAIA biofeedback training. Cappello et al. reported that the maximum reading speed was significantly improved after MH surgery [20]. Another group showed that the mean maximum reading speeds were comparable for eyes with closed MHs and their healthy fellow eyes [21]. Because we examined reading speed, fixation stability, and 63\% BCEA of the patients with successful closure of the $\mathrm{MH}$, a significant improvement was not detected even though all patients showed a tendency to improve their visual performance after MAIA biofeedback training. Tarita-Nistor et al. reported that change in fixation stability was a strong predictor of visual outcome after successful closure of the macular hole in 10 patients [22]. As shown in Table 2, patients 1 and 4 improved their fixation stability more than $14 \%$ by MAIA biofeedback training.

In patient 3, fixation stability and 63\% BCEA worsened after the training. It was possible that the point of PRT became inapt while retinal sensitivity was improved nonuniformly during the training. This case suggested that we should recalibrate PRT even during the training when fixation stability or $63 \%$ BCEA worsen.

Limitations of this study are the small sample size and the nonrandomized comparison. The effective number of training session and intervals between each training session are also unknown. However, most of the patients experienced an immediate improvement in visual performance after the first MAIA biofeedback training in this study. MAIA biofeedback training was easy for most patients and could be an option for patients whose recovery of visual acuity after successful MH surgery is insufficient.

Further studies with larger numbers of patients are needed to identify the most effective locations of the PRT and compare different methods of PRT location.

\section{Conflict of Interests}

None of the authors have any proprietary interests in this study.

\section{Acknowledgment}

The authors would like to express their gratitude for Toshihiko Oiwake's invaluable assistance with data analysis.

\section{References}

[1] N. E. Kelly and R. T. Wendel, "Vitreous surgery for idiopathic macular holes. Results of a pilot study," Archives of Ophthalmology, vol. 109, no. 5, pp. 654-659, 1991.

[2] D. W. Park, J. O. Sipperley, S. R. Sneed, P. U. Dugel, and J. Jacobsen, "Macular hole surgery with internal-limiting membrane peeling and intravitreous air," Ophthalmology, vol. 106, no. 7, pp. 1392-1398, 1999.

[3] H. L. Brooks Jr., "Macular hole surgery with and without internal limiting membrane peeling," Ophthalmology, vol. 107, no. 10, pp. 1939-1949, 2000.

[4] I. D. Fabian and J. Moisseiev, "Sutureless vitrectomy: evolution and current practices," British Journal of Ophthalmology, vol. 95, no. 3, pp. 318-324, 2011.

[5] S. Ullrich, C. Haritoglou, C. Gass, M. Schaumberger, M. W. Ulbig, and A. Kampik, "Macular hole size as a prognostic factor in macular hole surgery," British Journal of Ophthalmology, vol. 86, no. 4, pp. 390-393, 2002.

[6] L. A. Stec, R. D. Ross, G. A. Williams, M. T. Trese, R. R. Margherio, and M. S. Cox Jr., "Vitrectomy for chronic macular holes," Retina, vol. 24, no. 3, pp. 341-347, 2004.

[7] S. Kusuhara and A. Negi, "Predicting visual outcome following surgery for idiopathic macular holes," Ophthalmologica, vol. 231, no. 3, pp. 125-132, 2014.

[8] Z. Sun, D. Gan, C. Jiang et al., "Effect of preoperative retinal sensitivity and fixation on long-term prognosis for idiopathic 
macular holes," Graefe's Archive for Clinical and Experimental Ophthalmology, vol. 250, no. 11, pp. 1587-1596, 2012.

[9] J. M. Ruiz-Moreno, C. Staicu, D. P. Piñero, J. Montero, F. Lugo, and P. Amat, "Optical coherence tomography predictive factors for macular hole surgery outcome," British Journal of Ophthalmology, vol. 92, no. 5, pp. 640-644, 2008.

[10] K. Kawano, Y. Ito, M. Kondo et al., "Displacement of foveal area toward optic disc after macular hole surgery with internal limiting membrane peeling," Eye, vol. 27, no. 7, pp. 871-877, 2013.

[11] M. Ishida, Y. Ichikawa, R. Higashida, Y. Tsutsumi, A. Ishikawa, and Y. Imamura, "Retinal displacement toward optic disc after internal limiting membrane peeling for idiopathic macular hole," American Journal of Ophthalmology, vol. 157, no. 5, pp. 971-977, 2014.

[12] Y. Itoh, M. Inoue, T. Rii, Y. Ando, and A. Hirakata, "Asymmetrical recovery of cone outer segment tips line and foveal displacement after successful macular hole surgery," Investigative Ophthalmology \& Visual Science, vol. 55, no. 5, pp. 3003-3011, 2014.

[13] T. Nakagomi, T. Goto, Y. Tateno, T. Oshiro, and H. Iijima, "Macular slippage after macular hole surgery with internal limiting membrane peeling," Current Eye Research, vol. 38, no. 12, pp. 1255-1260, 2013.

[14] E. M. Vingolo, S. Cavarretta, D. Domanico, F. Parisi, and R. Malagola, "Microperimetric biofeedback in AMD patients," Applied Psychophysiology Biofeedback, vol. 32, no. 3-4, pp. 185189, 2007.

[15] E. M. Vingolo, S. Salvatore, and S. Cavarretta, "Low-vision rehabilitation by means of MP-1 biofeedback examination in patients with different macular diseases: a pilot study," Applied Psychophysiology Biofeedback, vol. 34, no. 2, pp. 127-133, 2009.

[16] M. D. Crossland, L. E. Culham, S. A. Kabanarou, and G. S. Rubin, "Preferred retinal locus development in patients with macular disease," Ophthalmology, vol. 112, no. 9, pp. 1579-1585, 2005.

[17] C. Buia and P. Tiesinga, "Attentional modulation of firing rate and synchrony in a model cortical network," Journal of Computational Neuroscience, vol. 20, no. 3, pp. 247-264, 2006.

[18] E. Altpeter, M. Mackeben, and S. Trauzettel-Klosinski, "The importance of sustained attention for patients with maculopathies," Vision Research, vol. 40, no. 10-12, pp. 1539-1547, 2000.

[19] H. Ozdemir, M. Karacorlu, F. Senturk, S. A. Karacorlu, and O. Uysal, "Retinal sensitivity and fixation changes 1 year after triamcinolone acetonide assisted internal limiting membrane peeling for macular hole surgery-a MP-1 microperimetric study," Acta Ophthalmologica, vol. 88, no. 6, pp. e222-e227, 2010.

[20] E. Cappello, G. Virgili, L. Tollot, M. D. Borrello, U. Menchini, and M. Zemella, "Reading ability and retinal sensitivity after surgery for macular hole and macular pucker," Retina, vol. 29, no. 8, pp. 1111-1118, 2009.

[21] S. Richter-Mueksch, S. Sacu, E. Osarovsky-Sasin, E. Stifter, C. Kiss, and M. Velikay-Parel, "Visual performance 3 years after successful macular hole surgery," British Journal of Ophthalmology, vol. 93, no. 5, pp. 660-663, 2009.

[22] L. Tarita-Nistor, E. G. González, M. S. Mandelcorn, L. Lillakas, and M. J. Steinbach, "Fixation stability, fixation location, and visual acuity after successful macular hole surgery," Investigative Ophthalmology \& Visual Science, vol. 50, no. 1, pp. 84-89, 2009. 


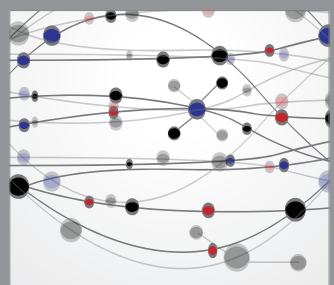

The Scientific World Journal
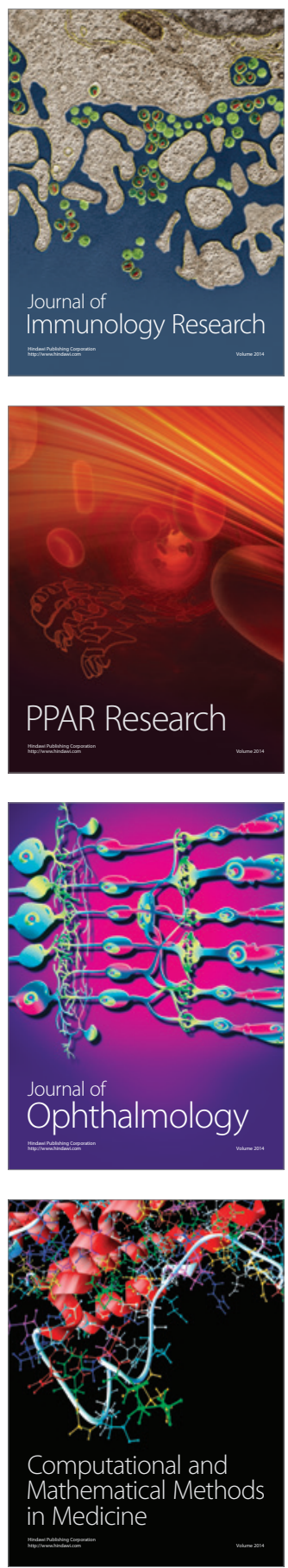

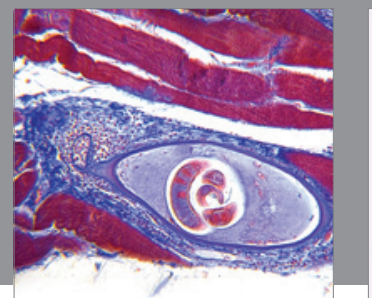

Gastroenterology

Research and Practice
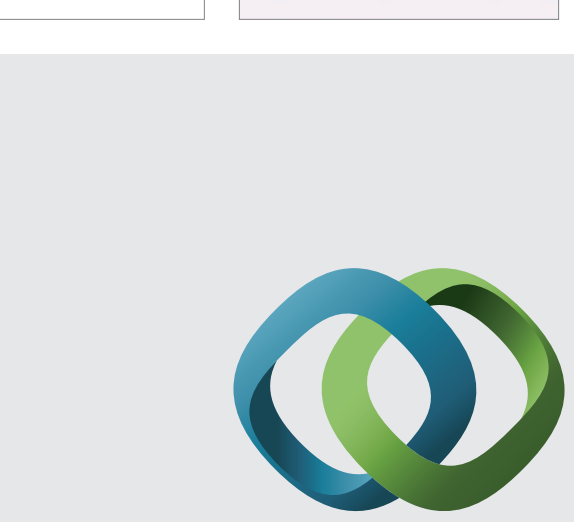

\section{Hindawi}

Submit your manuscripts at

http://www.hindawi.com
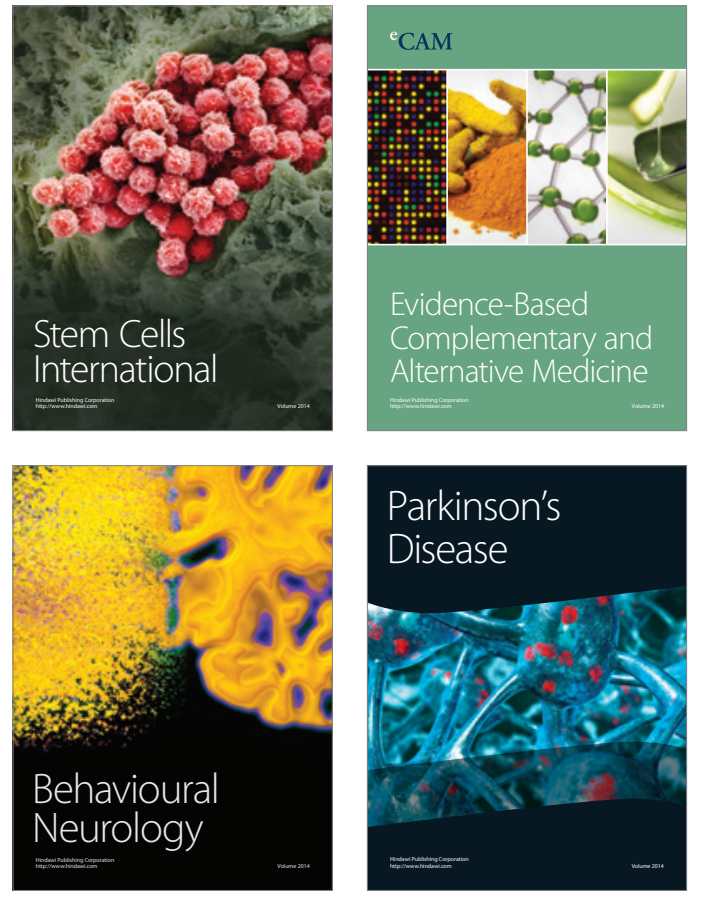
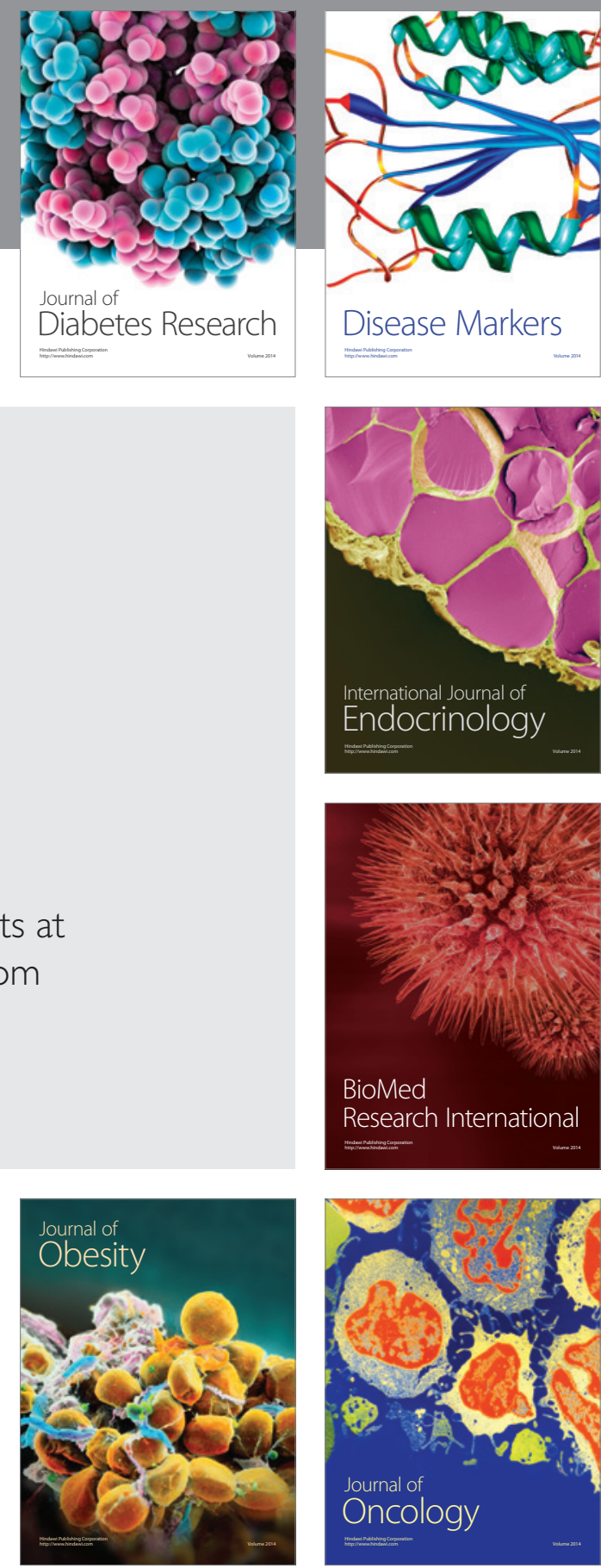

Disease Markers
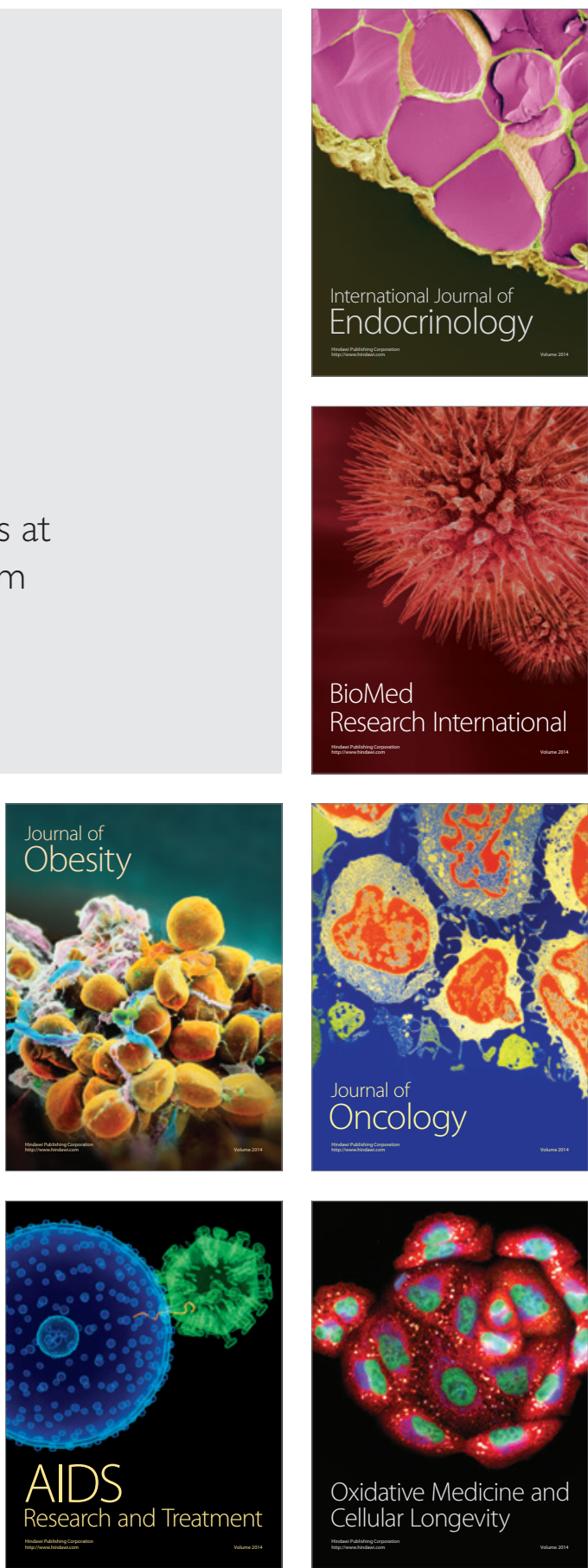